Meeting Abstracts

ma.ecsdl.org

Abstract MA2016-01 1848

\title{
Micro Computed Tomography As Powerful Tool for Analyzing Post Mortem Biofilm and Carbonate on Operated Cathode in Single Chamber Microbial Fuel Cell
}

\author{
Maurizio Santini ${ }^{a}$, Manfredo Guilizzoni ${ }^{b}$, Massimo Lorenzi ${ }^{a}$, Plamen Atanassov ${ }^{c}$, \\ Enrico Marsili $^{d}$, Stephanie Fest-Santini ${ }^{a}$, Pierangela Cristiani ${ }^{e}$ and Carlo Santoro ${ }^{f}$ \\ Author Affiliations
}

\begin{abstract}
The cathode performances are one of the main limitations related with microbial fuel cell (MFC) that strongly limit the overall output. The low power produced is an important problem that has to be addressed for the scale up of the MFCs.

Cathodic overpotential is particularly high at circumneutral $\mathrm{pH}$ when carbonaceous or metallic catalysts are used [1]. Overpotentials are much lower for enzymes but enzymatic biocathodes are expensive and not durable [2]. Single chamber membrane-less MFCs are preferred due to the lower cost and higher power output. In membrane-less MFCs, both electrodes (anode and cathode) are exposed directly to the electrolyte or carbon-rich solution. The presence of growing bacteria inside the chamber generates electron acceptor-rich and electron donor-rich zones, which serve as cathode and anode, respectively. In fact, bacteria consume rapidly the oxygen inside the chamber creating anaerobic conditions that are useful for electrogenic bacteria for colonizing the anode electrode and oxidize organics. The cathode used is an air-breathing cathode and it is porous and allows oxygen to penetrate through the anodic solution. A biofilm is formed also on the cathode surface consuming the oxygen and forming a natural and cheap membrane that separate the cathode from the solution and stop the oxygen leaking inside the chamber, thus ensuring anaerobic conditions. Strict anaerobes grow quickly within 1 week of operations as recently showed [3]. It has been showed previously that the biofilm formation on the cathode initially increased the cathodic reduction reaction [4].
\end{abstract}

In this work, we analyzed the cathode performances and the MFC overall performance along 6 months operations [5]. At the end of the experimentation, the cathode has been examined with X-Ray Micro Computed Tomography (microCT), scanning electron microscopy (SEM), and energy-dispersive X-ray spectroscopy (EDX) for characterizing the organic/inorganic layer on the cathode surface facing the solution [5]. Single chamber MFC with volume of $125 \mathrm{~mL}$ was used and the cell was fed with raw wastewater and sodium acetate. The results obtained showed that cathode reduction reaction increased in the first 15 days but then decreased continuously over time [5]. The initial increase might be due to the formation of an initial biofilm layer that probably enhanced the cathodic reduction reaction. Over time, precipitation of compounds and fouling might take place. It has been speculated that thick biofouling decreased the 
performances.

After 6 months, part of the cathode was dried and analyzed with SEM/EDX and the other part was analyzed as it is using microCT [5]. SEM has the great potential of very high resolution but images have to be taken after treating the sample and consequently change the morphology of the biofilm after drying it. Moreover, SEM is not able to pass through objects and consequently only the surface can be analyzed. The coupling with EDX allowed to identify sodium ( $\mathrm{Na}$ ) on the external part of the cathode and calcium (Ca) on the internal face [5]. Na and Ca were present in form of carbonate.

The production of $\mathrm{OH}$ - due to the cathodic reaction, caused the alkalinization of solution at the interface with the cathode making the precipitation of carbonate species on the cathode is very probable. MicroCT images allowed making a complete 3D scan of the entire cathode analyzed. Interestingly, the different components (cathode, carbonate and biofilm) were clearly distinguishable (Figure 1). In fact, a compact and continuous thick layer of carbonate was clearly visible between the cathode and the biofilm (Figure 1 , white color). Those results obtained using microCT clearly showed that the thick, not porous carbonate layer and not the biofilm itself might increase the proton mass transport resistance from the solution to the catalytic sites and lowering dramatically the cathode output during long terms operation.

\section{Reference}

[1] C. Santoro, F. Soavi, A. Serov, C. Arbizzani, P. Atanassov. Biosensors and Bioelectronics. 2016, 78, 229-235.

[2] C. Santoro, S. Babanova, B. Erable, A. Schuler, P. Atanassov. Bioelectrochemistry. 2016, 108, 1-7.

[3] M. Grattieri, S. Babanova, C. Santoro, E. Guerrini, S.P.M. Trasatti, P. Cristiani, M. Bestetti, P. Atanassov. Electroanalysis, 2015, 27(2), 327-335.

[4] P. Cristiani, M.L. Carvalho, E. Guerrini, M. Daghio, C. Santoro, B. Li. Bioelectrochemistry, 2013, 92, 6-13.

[5] M. Santini, M. Guilizzoni, M. Lorenzi, P. Atanassov, E. Marsili, S. Fest-Santini, P. Cristiani, C. Santoro. Biointerphases. 2015, 10, 031009.

Figure 1. Cross Section of the cathode analyzed using microCT.

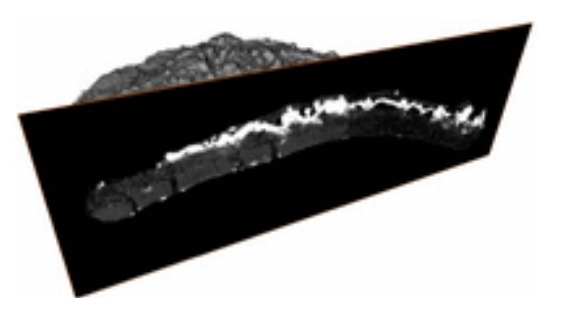

View larger version: In this page In a new window Download as PowerPoint Slide
Figure 1

(c) 2016 ECS - The Electrochemical Society 